\title{
Destabilization of Hydrophobic Core of Chicken Villin Headpiece in Guanidinium Chloride Induced Denaturation: Hint of $\pi$-cation Interaction
}

\author{
Sridip Parui, Rabindra Nath Manna, Biman Jana*
}

Department of Physical Chemistry, Indian Association for the Cultivation of Science, Jadavpur, Kolkata 700032 , India

\section{Hydrogen bond and native contacts}

In our calculation, the hydrogen bond is considered to be present if the distance between the donor (D) and the acceptor (A) is $<=3.5 \AA$ and the angle $\mathrm{D}-\mathrm{H}---\mathrm{A}$ is $>=140 \mathrm{deg}$. A native contact between two atoms is regarded to be present if the distance between them is less than 1.2 times the native distance in the crystal structure.

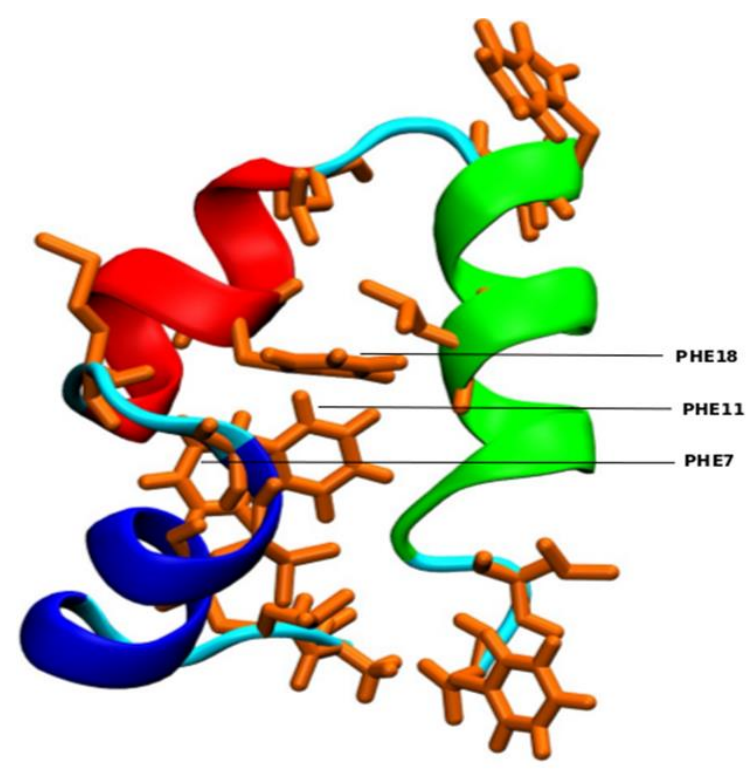

Figure S1. Native structure of HP-36 


\section{Steered Molecular Dynamics}

a)

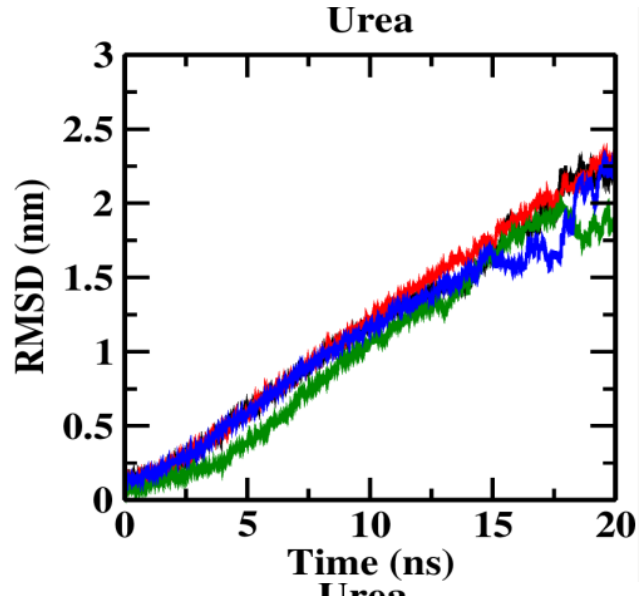

b)

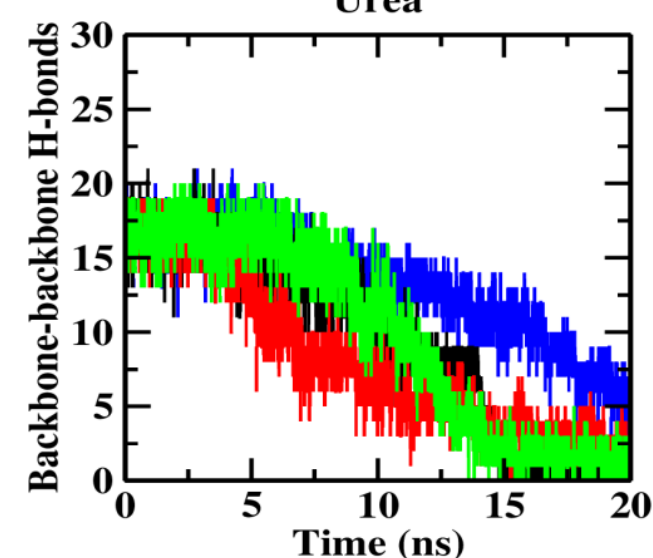

c)

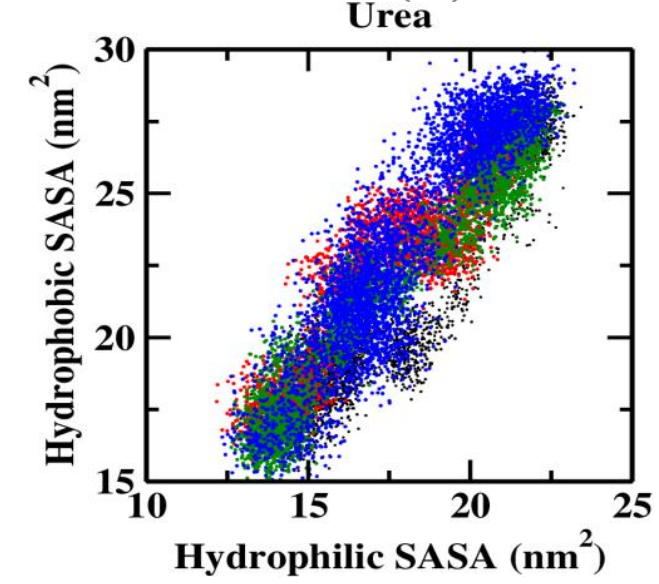

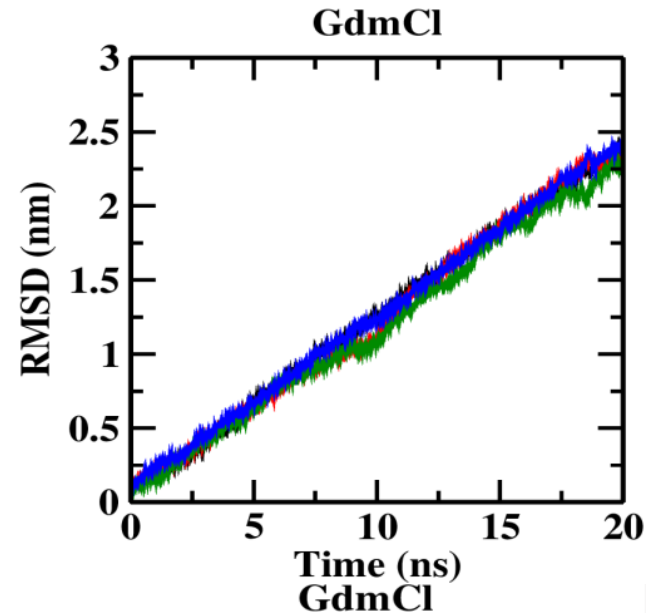
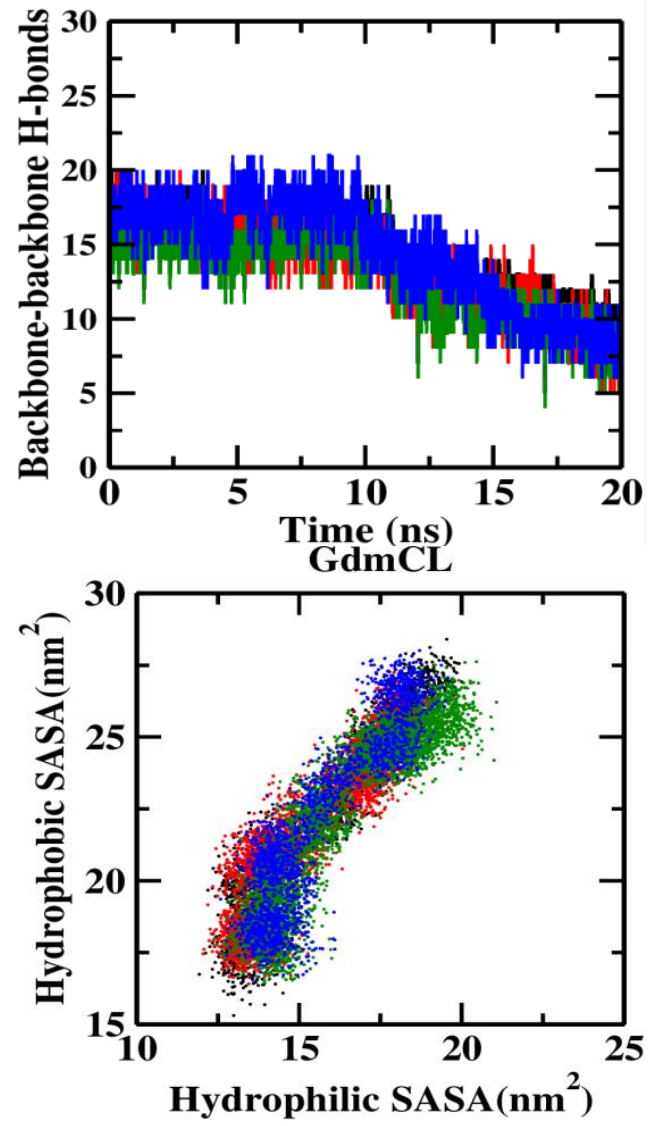

Figure S2. Time evolution of (a) RMSD from native state, (b) number of backbone-backbone $\mathrm{H}$-bonds during the unfolding of HP-36. (c) Unfolding trajectories are shown in a two dimensional plot based on hydrophilic SASA and hydrophilic SASA. In each denaturant condition, four simulations have been performed and are represented by different colors. 
a)

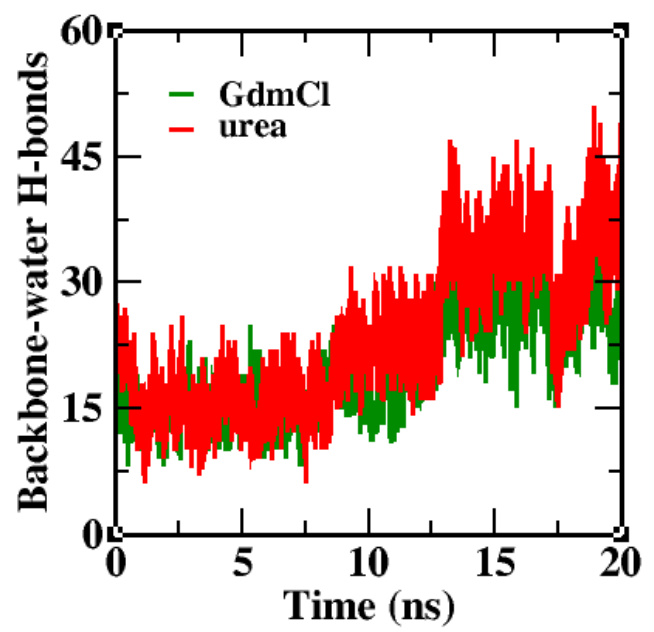

b)

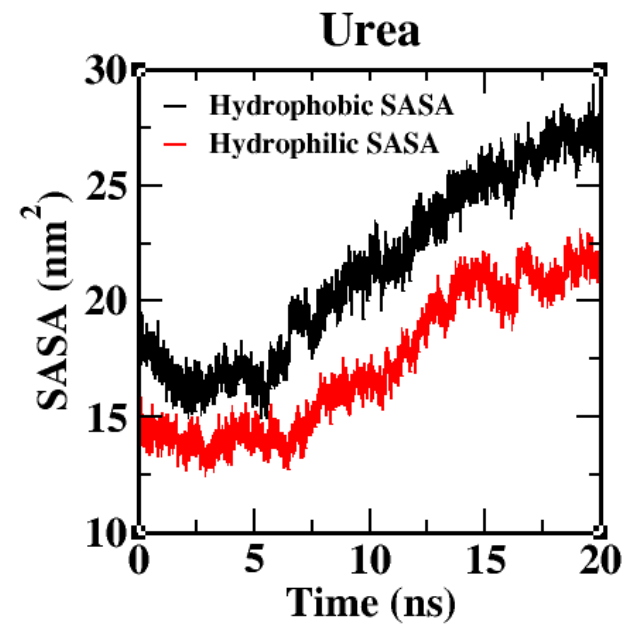

\section{GdmCl}

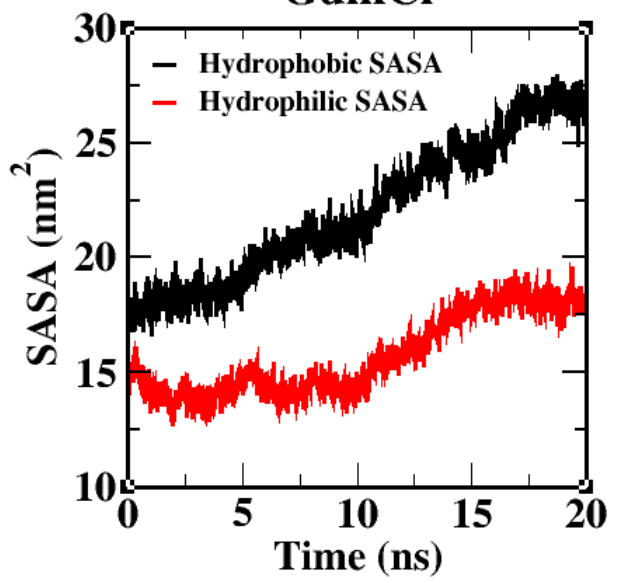

Figure S3. Time evolution of (a) number of backbone-water H-bonds, (b) hydrophilic and hydrophobic SASA during unfolding. 
a)

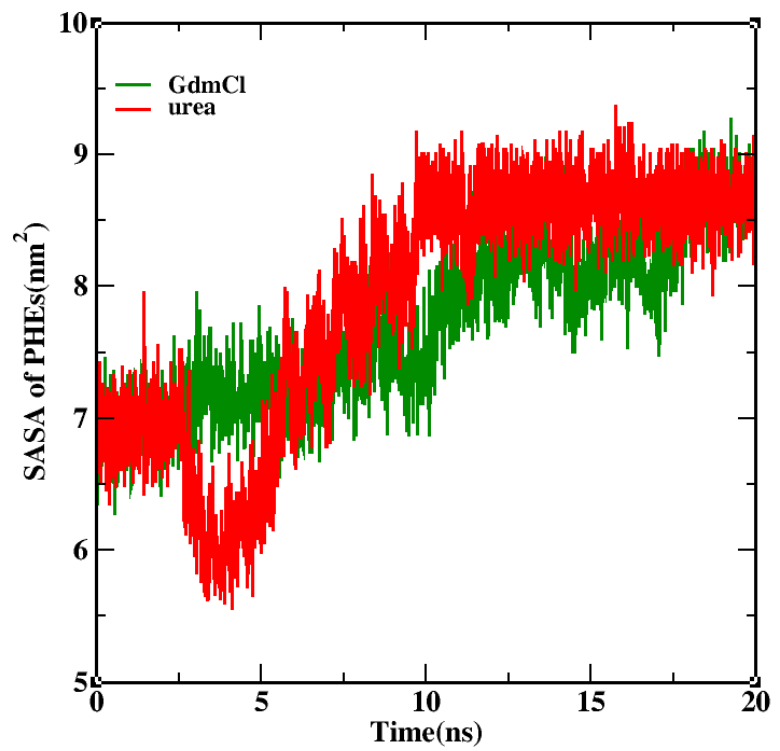

c)

b)
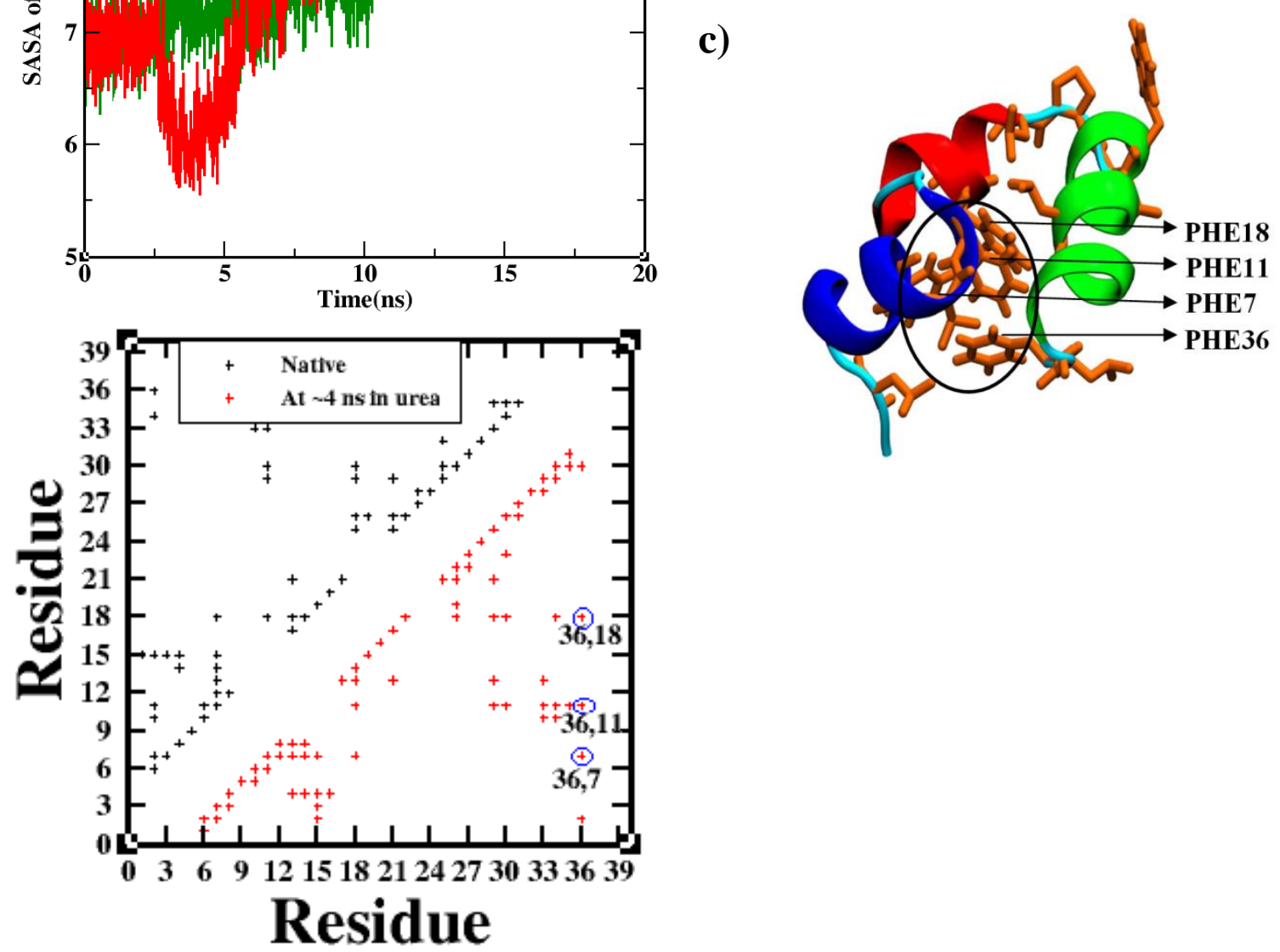

Figure S4. (a) Time evolution of SASA of side chain aromatic residues during unfolding. (b) Contact map of HP-36 at native and compact states(a frame obatined $\sim 4$ ns of urea induced unfolding). (c) a representative snapshot of compact state. 
a)

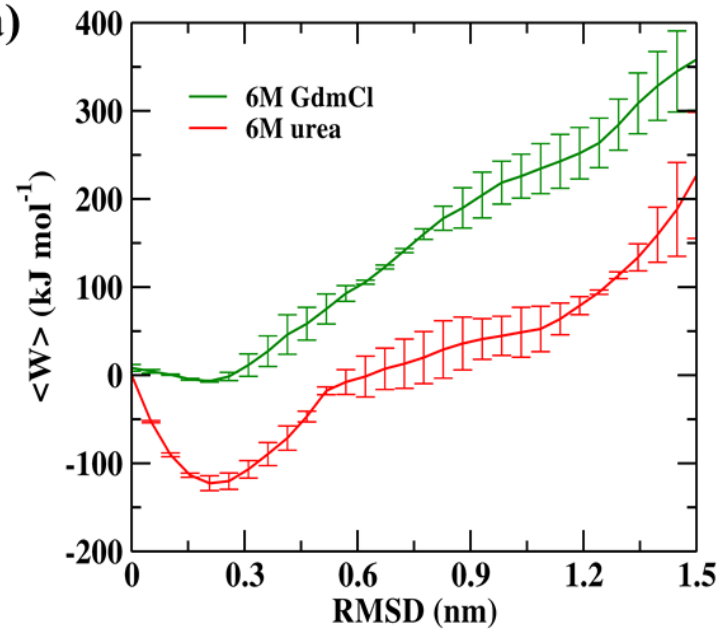

b)

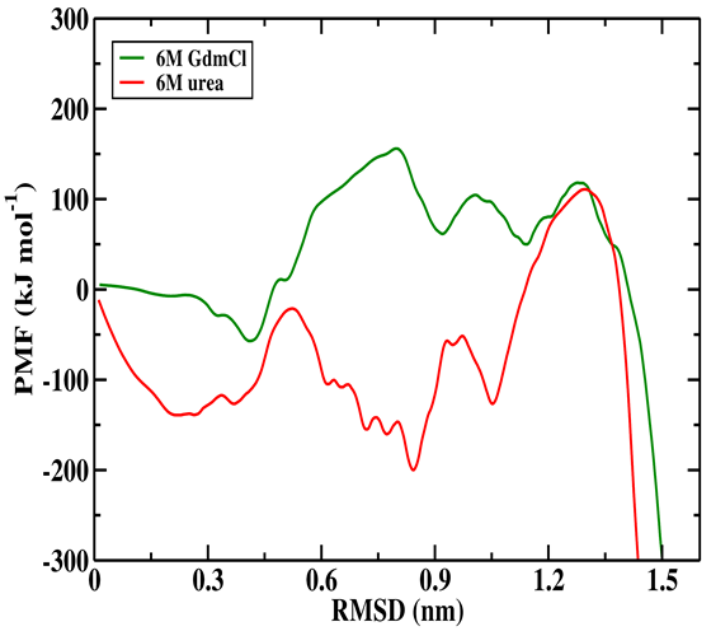

Figure S5. (a) Average work profile with standard deviation as a function of RMSD during the unfolding of the SMD runs. (b) PMF calculation using the second order cumulant expansion of the irreversible work (Jarzinsky's Equality). 


\section{Umbrella sampling}

a)

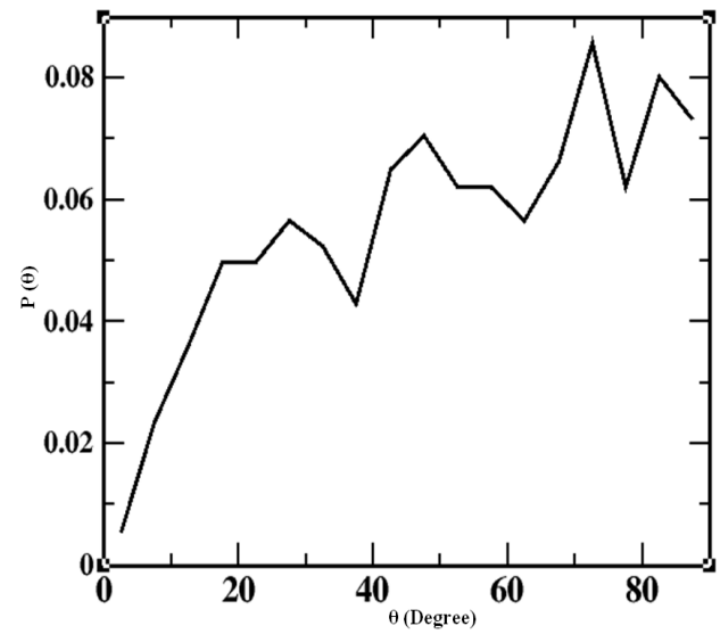

b)

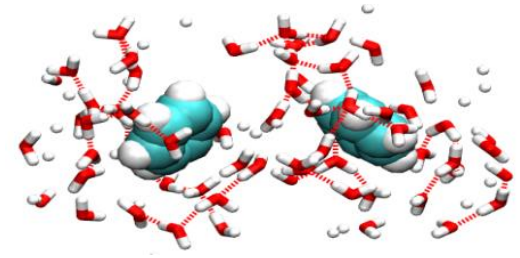

c)

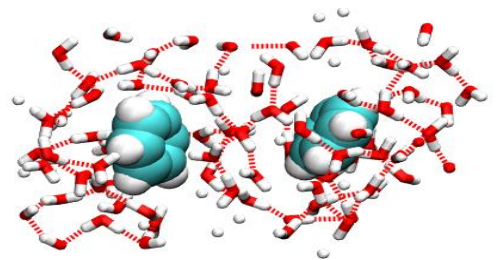

d)

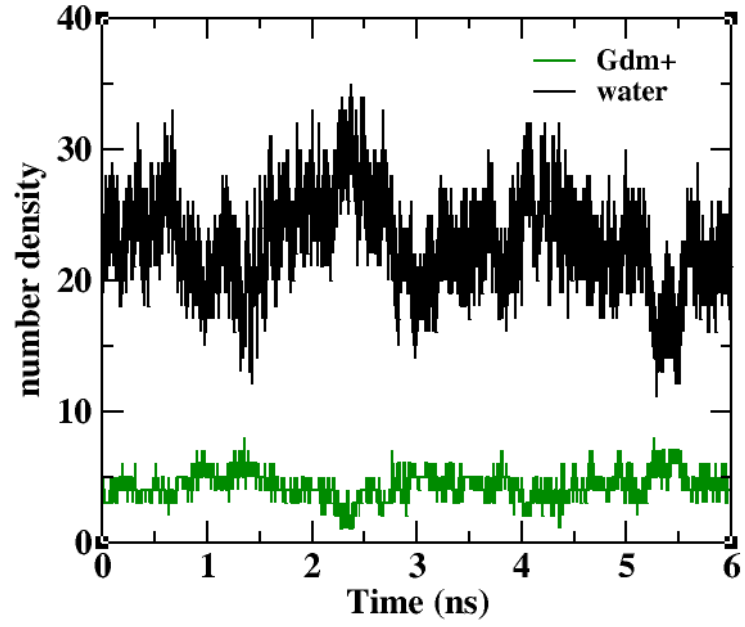

Figure S6. (a) Distribution of angle ( $\theta$ ) between the plane of benzene and the plane of urea at the SSM. Arrangement of water around benzenes at solvent separated minima in $\mathrm{GdmCl}$ (b) and in urea (c). Note the comparatively more disrupted water network for $\mathrm{GdmCl}$ than urea. (d) Number density of water and guanidinium cation around $0.6 \mathrm{~nm}$ of benzene. 


\section{Standard Molecular dynamics simulation at 400K}
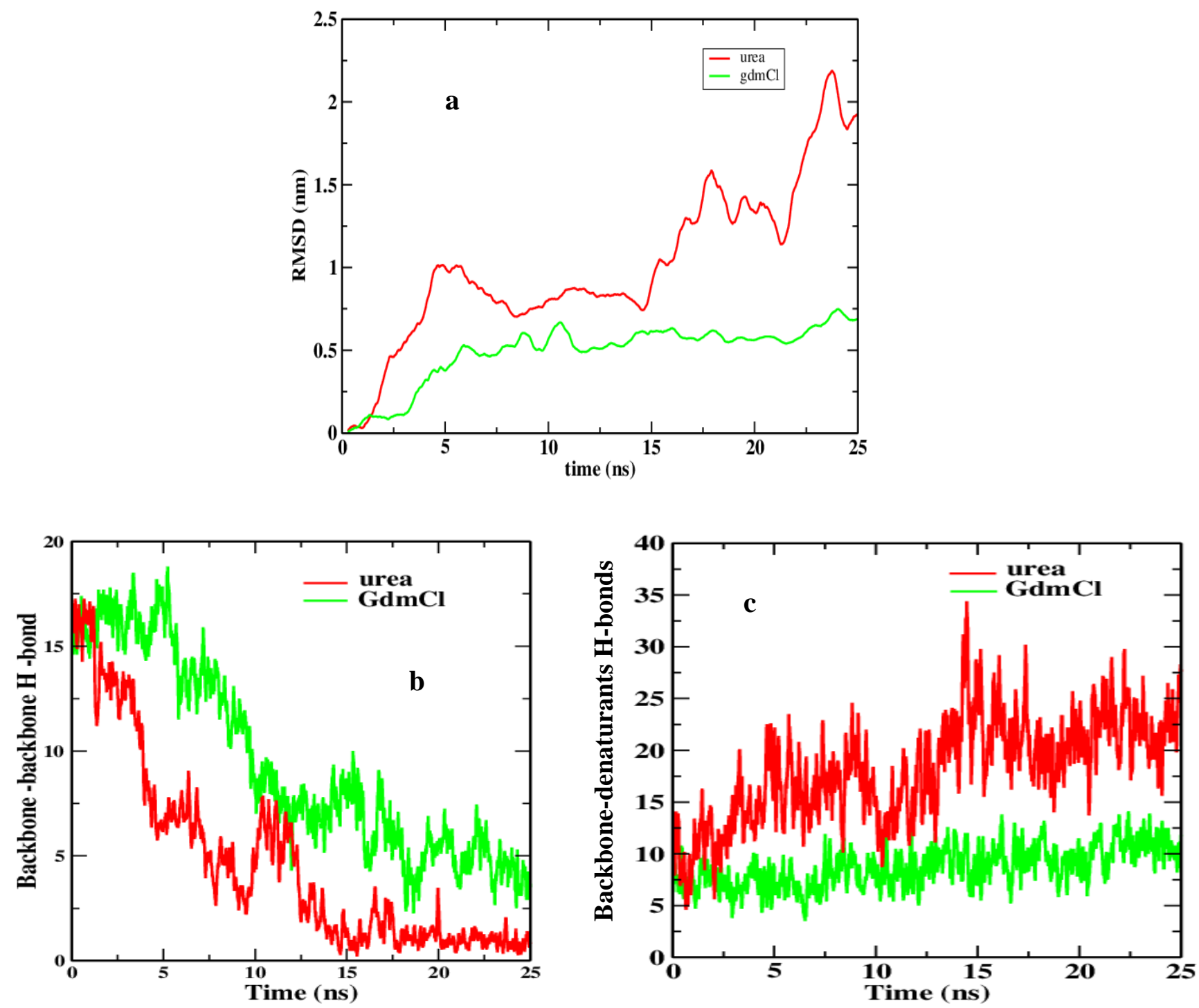

Figure S7. Time evolution of (a) RMSD from native state, (b) number of backbone-backbone Hbonds and (c) number of backbone-denaturants H-bondsduring the course of unfolding. 